\title{
0359 OUALITY OF CHILD ABUSE INFORMATION FOR MEASUREMENT AND SURVEILLANCE: LINKING HOSPITAL AND CHILD WELFARE DATA
}

K McKenzie*, D Scott, J Fraser, M Dunne Correspondence: Queensland University of Technology, Victoria Park Rd, Kelvin Grove, Queensland 4059, Australia

10.1136/ip.2010.029215.359

National estimates of the prevalence of child abuse-related injuries are obtained from a variety of sectors including welfare, justice and health resulting in inconsistent estimates across sectors. The International Classification of Diseases (ICD) is used as the international standard for categorising health data and aggregating data for statistical purposes, though there has been limited validation of the quality, completeness or concordance of these data with other sectors. This research study examined the quality of documentation and coding of child abuse recorded in hospital records in Queensland and the concordance of these data with child welfare records. A retrospective medical record review was used to examine the clinical documentation of over 1000 hospitalised injured children from 20 hospitals in Queensland. A data linkage methodology was used to link these records with records in the child welfare database. Cases were sampled from three sub-groups according to the presence of target ICD codes: Definite abuse, Possible abuse, unintentional injury. Less than $2 \%$ of cases coded as being unintentional were recoded after review as being possible abuse, and only $5 \%$ of cases coded as possible abuse cases were reclassified as unintentional, though there was greater variation in the classification of cases as definite abuse compared to possible abuse. Concordance of health data with child welfare data varied across patient subgroups. This study will inform the development of strategies to improve the quality, consistency and concordance of information between health and welfare agencies to ensure adequate system responses to children at risk of abuse. 